Endocrinol. Japon. 1963, 10 (4), 215 220

\title{
CONGENITAL ADRENAL HYPERPLASIA ASSOCIATED WITH GONADAL AGENESIS*
}

\author{
NOBUAKI SASANO, MICHIKO FURUYAMA** \\ AND MASAYOSHI YAMAZAKI
}

First Institute of Pathology, Tohoku University

School of Medicine, Sendai

Congenital adrenal hyperplasia is usually combined with the picture of congenital adrenogenital syndrome due to increased secretion of androgenic steroids. According to the review of 135 cases of this disease by Iversen (1955), 75 out of 78 female cases presented pseudohermaphroditism with hypertrophy of the clitoris and a common urethral and vaginal orifice. In 16 out of 57 boys had macrogenitosomia praecox, while hypospadias (male pseudohermaphroditism) was observed in 6 cases. The combination of congenital adrenocortical hyperplasia with severe developmental failure of the internal genitalia and the normal external female ones as will be demonstrated in this paper as gonadal agenesis, has not been appeared in the literature listed by Iversen (1955) or reported after him. The significance of interesting morphologic findings in the pituitary and the adrenal glands will be discussed in comparison with those of the congenital adrenal hyperplasia with male pseudohermaphroditism.

\section{CASE REPORT}

\section{Clinical Record}

A 13-days-old apparent female newborn was the second baby of healthy parents. The first baby of them died of vomiting and general weakness, with the same symptoms as in the present patient, on the 30th neonatal day.

During the course of pregnancy, the mother had no abnormalities and never received a hormone administration. The patient was born on October 22nd, 1960 in a hospital as a mature infant weighing $3900 \mathrm{~g}$. Cyanosis particularly notable on the face and vomiting 3 to 6 times a day as well appeared soon after the birth and persisted throughout the clinical course of the patient. Jaundice appeared on the 4th day and diminished after a week. General weakness was followed by tachypnea and pneumonia was suspected although the patient did not show fever. Administration of antibiotics was ineffective and the patient died on November 4th, 1960, when the body weight decreased to $3620 \mathrm{~g}$.

Postmortem Diagnosis (No. 259-60)

1. Congenital adrenocortical hyperplasia, bilateral, severe.

2. Gonadal agenesis without development of adnexa uteri.

3. Desquamative pneumonia of both lungs, acute, extensive.

Received for publication November 8, 1963.

* Supported by the Grant in Aid for Fundamental Scientific Research of the Ministry of Education (710500 in 1963).

**Dr. Furuyama's present address is Prof. Torikai's Department of Internal Medicine, Tohoku University School of Medicine. 


\section{Autopsy Findings}

A well-developed newborn had no abnormalities on the external surface of the body and extremities except for slight icterus. The external genitalia including vagina and urethra were entirely normal for a newborn female while notable malformations were observed in the internal genitalia and the ovaries and the Fallopian tubes could not be detected on both sides. The uterus showed evident dysplasia forming an elliptic mass $(12 \times 6 \times 6 \mathrm{~mm})$ of musculofibrous tissue behind the cervical region of the urinary bladder without grossly detectable lumen. The tissues in the genital regions were cut into several slices for histological examinations and the following two kinds of glandular structures were histologically confirmed in the mass of the uterine region. The one observed in the central part of the mass was composed of a layer of multifold columnar epithelium with relatively well developed lumen suggesting the uterine cavity. The other in the posterio-lateral region was composed of tubular tissues lined by a layer of ciliated epithelium suggesting the rest of Gartner's duct. No tissues suggesting the ovary or testis were found. The epithelial cells of the liver and other organs showed a chromatin negative pattern, although the external genitalia were of female type.

The adrenal glands were distinctly enlarged weighing $12 \mathrm{~g}$ on the left side and $12.5 \mathrm{~g}$ on the right side. The cut surface showed diffuse thickening of the cortex with partial nodulation. Pale brown-yellow color was observed in the periphery and hyperemia in the central part. Diffuse and adenomatous hyperplasia of the cortical cells was histologically observed throughout the glands, being composed of the clearly swollen cells with the foamy or spongy protoplasm and the eccentric, small nuclei frequently exhibiting pycnosis. Small foci of the compact cells rarely associated with the hypertrophic nuclei were occasionally found and has a transition from the above clear cells. Frozen section revealed a lot of birefringent crystaline materials in the clear cells of hyperplastic cortex. Small foci containing the needle-like giant crystals and the multinuclear giant cells were occasionally found together with granulomatous lesions. Fine droplets of sudanophil materials were found in various amounts within both clear and compact cells but the birefringent crystals were densely observed in the clear cells even without sudanophil droplets. Histochemical methods for cholesterol by Schultz (1924) and Okamoto et al. (1944) gave almost negative reactions. In the subcapsular regions, somewhat hypertrophic compact cells with small amount of fine lipid droplets were prevalent and the normal structure of the glomerular zone containing small clear cells could not be detected. No particular abnormalities were found in the medulla.

The anterior pituitary had distinct hyperplasia and hypertrophy of the well-granulated and the degranulated basophils and the cells ambiguously stained with PAS-orange G and paraldehydefuchsine Goldner methods. Several areas of localized adenomatous hyperplasia of the basophils comparable to microadenoma were observed and occasionally accompanied by the glandular lumen containing colloid material, with positive PAS stain.

The lungs had moderate desquamative pneumonia with hyperemia and edema. Signs of aspiration of amniotic fluid were also confirmed. Slight acute splenitis and the initial stage of thymic involution were additional findings.

\section{DISCUSSION}

In usual cases of congenital adrenal hyperplasia with pseudohermaphroditism, the sex differentiation is entirely female in genetics as well as in the internal genitalia; i.e., the ovaries, Fallopian tubes and uterus are generally normal, while the phallus and labioscrotal folds show various degrees of abnormalities. This is generally explained by the difference of the stage of the differentiation of internal and external genital organs at which the androgenic effects of adrenal hyper- 


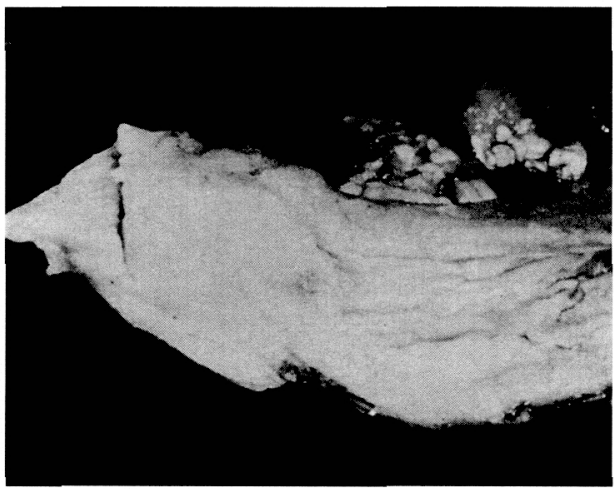

Fig. 1. A small mass of fibro-muscular tissue in the region of uterine cervix

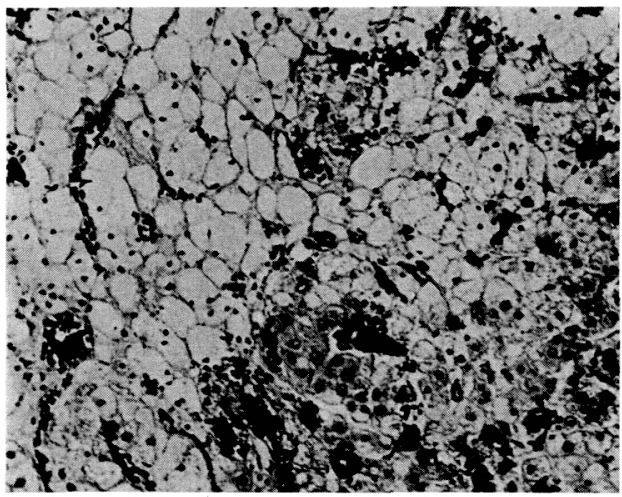

Fig. 3. Hyperplasia of the spongy cortical cell with a transition to the compact cell hematoxyline and eosin $\times 100$

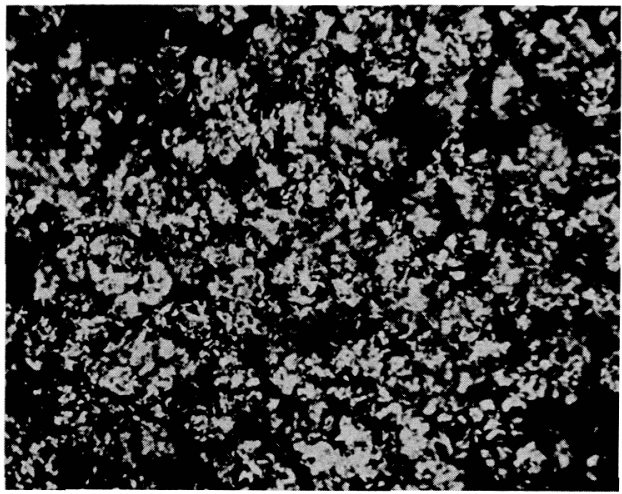

Fig. 5. The crystal gives a distinct birefringency. $\times 200$

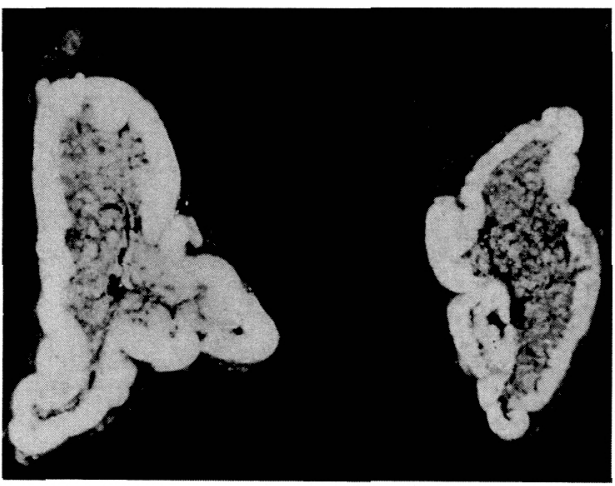

Fig. 2. Hyperplasia of the adrenal cortex

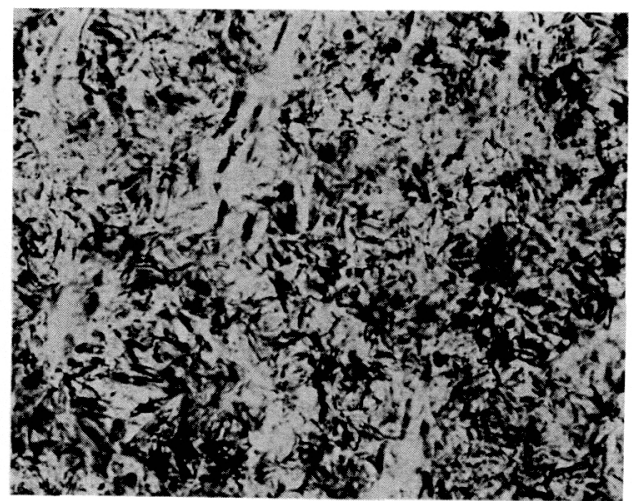

Fig. 4. The spongy cells are filled with needle-like crystaline materials. Sudan III $\times 250$

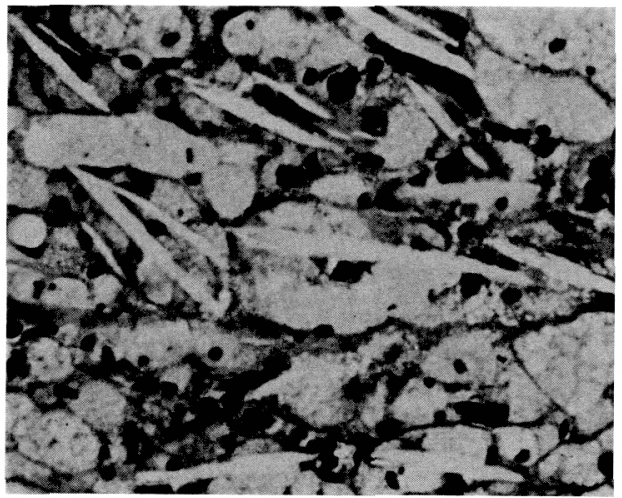

Fig. 6. A lesion containing large clystaline clefts within the hyperplasia hematoxyline and eosin $\times 420$ 


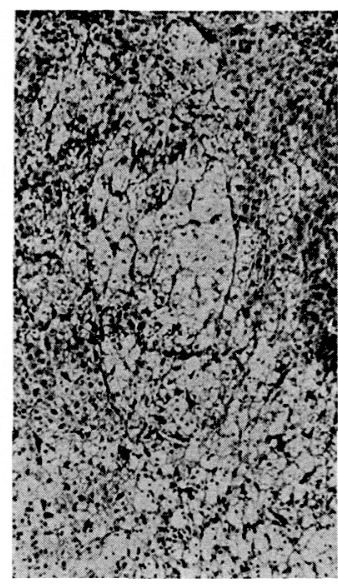

Fig. 7. Hyperplasia of the deeper cortex sometimes extends toward the subcapsular regions.

hematoxyline and eosin $\times 60$

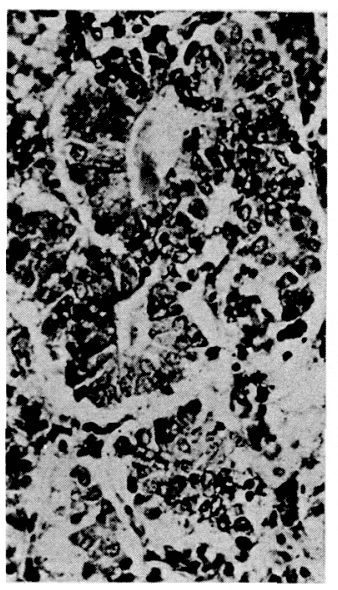

Fig. 8. Distinct hyperplasia of basophils in the anterior pituitary exhibits a glandular arrange. ment.

hematoxyline and eosin $\times 250$

plasia become manifest. In the present case, the defects of Müllerian ducts probably existed before excessive androgen secretion. It is regretted that the chromosomal determination was not performed on this patient although it was suggested that she was most likely XO as Ford et al. (1959) and Kumamoto (1961) discussed.

A considerable proportion of patients with gonadal agenesis are chromatinnegative, although chromatin-negativity is an invariable feature of normal males (Ford et al., 1959). This is accounted for by the experimental results that bilateral castration of the fetus of both sex in an early undifferentiated stage resulted in completely feminine type of internal and external genital organs (Polani et al., 1954; Willis, 1958; Kumamoto 1961). It is beyond question that the defect of Müllerian duct observed in the present case includes ovarian agenesis or gonadal aplasia.

The histology of the hyperplastic adrenal glands in the present case was of further interest. Congenital adrenal hyperplasia is usually composed of eosinophilic compact cells with finely granular cytoplasm containing fine lipid droplets and the findings are comparable to those of an ACTH-treated gland (Symington and Jeffries, 1962). Blackman (1946) observed that the postnatal reticular zone was hyperplastic even in newborn and infant pseudohermaphrodites and Jones and Jones (1954) supported his opinion. Seelen (1960) observed that the fascicular zone was relatively well preserved, while the width of the reticular zone increased. The description of these authors represented by hyperplasia of compact cells are based on usual cases of congenital adrenal hyperplasia with androgenic activities. On the other hand, the cases with feminization in male are entirely rare but have a little different adrenal histology from that in cases with masculini- 
zation. Dohm (1958) reported a newborn male pseudohermaphrodite associated with congenital adrenal hyperplasia consisting of large vacuolar cells without lipid deposition. The external genitalia simulated the female but the testes were situated in the labial folds. Gurtner (1955) presented a case with the same combination and observed that the adrenocortical hyperplasia was mainly composed of spongiocytes containing numerous birefringent crystals and sporadically of multinuclear giant cells with and without large needle-like crystals. In the present case no testicular tissues were confirmed in the labial folds or abdomen, however similar findings to those of the above cited cases of congenital adrenal hyperplasia with male pseudohermaphroditism were confirmed. It is generally accepted that administration of estrogenic hormones during fetal life may induce adrenal hyperplasia and reversion of the gonads, and that an abnormal maternal estrogenic influence on the fetus may be a probable cause of the congenital adrenogenital syndrome in man (Iversen, 1955). The mother of the present case did not receive an administration of estrogenic or progesterous hormone during the pregnancy. Therefore, it is reasonable to consider that the hyperplastic cortical cells may produce estrogenic hormones although actual evidence could not be presented.

The anterior pituitary of the most patients with congenital adrenal hyperplasia usually exhibits a definite or possible increase in basophils or an increased number of hyaline basophilic cells and hypertrophic amphophils (Mellgren, 1945; Iversen, 1955; Russfield, 1957). Moderate hyperplasia of amphophils is also confirmed in patients with congenital gonadal agenesis, while true acidophils were within normal limits (Russfield, 1957). A distinct hyperplasia in the basophils simulating multiple microadenomas was confirmed in the present case and this would be significant to consider that the case had both congenital adrenal hyperplasia and a severe form of gonadal agenesis.

\section{SUMMARY}

A rare autopsy case of congenital adrenal hyperplasia combined with agenesis of the gonad and adnexa was reported. The patient was a newborn with negative sex chromatin and the normal external female genitalia. The hyperplastic adrenocortical tissues were generally composed of spongy cells with tremendous accumulation of birefringent crystaline materials. Small lesions containing large crystaline clefts occasionally surrounded by a granulomatous cell-infiltration were additional findings. The adrenal findings were different from those of usual congenital adrenal hyperplasia but similar to those of the case with male pseudohermaphroditism reported by Gurtner (1955). Multifocal adenomatous hyperplasia of the basophils was evident in the anterior pituitary. 


\section{REFERENCES}

Blackman, S.S. (1946). Bull. Johns Hopkins Hosp. 78, 180.

Dhom, G. (1958). Zbl. Path. 97, 346.

Ford, C.E., K.W. Jones, P.E. Polani, J.C. de Almeida and J.H. Briggs (1959). Lancet 1, 771. Gurtner, H.P. (1955). Virchow's Arch. Pathol. Anat. u Physiol. 326, 409.

Iversen, T. (1955). Pediatrics 16, 875.

Jones, H.W. and G.E.S. Jones (1954). Am. J. Obstet. Gynecol. 68, 1330.

Kumamoto, E. (1961). Nihonrinsho 19, 2251. (In Japanese)

Mellgren, J. (1945). Acta Pathol. Microbiol. Scand. Supple. 60, 1.

Okamoto, K., H. Shimamoto and H. Sonoda (1944). Jap. J. Constit. Med. 13, 113. (In Japanese) Polani, P.E., W.F. Hunter and B. Lennox (1954). Lancet 2, 120.

Russfield, A.G. The Adenohypophysis; Mellor's Analytical Pathology. Blakiston, New York, p. 293 (1957).

Schultz, A. (1924). Zbl. Path. 35, 314.

Seelen, J.C. (1960). Acta Endocrinol. 34, 457.

Symington, T. and R. Jeffries. The Pathology of Adrenocortical Lesions Causing Hyperadrenalism; Currie and Others' Adrenal Cortex. Livingstone, Edinburgh, p. 345 (1962).

Willis, R.A. The Borderland of Embryology and Pathology. Butterworth and Co., London, p. 204 (1958). 